\title{
PATENT DUCTUS ARTERIOSUS
}

\section{A REVIEW OF 108 CASES TREATED SURGICALLY}

\author{
BY \\ J. D. HAY and O. C. WARD \\ From the Royal Liverpool Children's Hospital
}

(RECEIVED FOR PUBLICATION APRIL 5, 1956)

Surgical closure of a patent ductus arteriosus was first reported by Gross and Hubbard in the year 1939. Since then the procedure has been attempted in thousands of cases with a mortality rate in the larger published series of from 0 to $2 \%$ (Gilchrist, 1948; Sellors, 1948; Potts, Gibson, Smith and Riker, 1949; Scott, 1950; Gross, 1952; Starer, 1953; Tubbs, 1955). Simple ligature of the ductus has in some cases failed to achieve complete obliteration of its lumen and in others has been followed by recanalization. Gross therefore in 1947 adopted the practice of dividing the vessel and many surgeons now follow his example, especially when dealing with a large ductus.

We have reviewed the first 108 children between the ages of 1 and 16 years referred to one of us (J.D.H.) with a patent ductus arteriosus and treated surgically by Mr. F. Ronald Edwards and Mr. B. J. Bickford, and have attempted to assess not only the results of operation and their bearing upon the choice of surgical procedure, but also the value of the various diagnostic criteria of this condition. We have excluded infants under the age of 1 year because they present special problems in diagnosis and treatment.

The patients were referred by other consultants, school medical officers and family doctors and are probably representative of children seen in other centres with patent ductus arteriosus during the period under review, 1949 to 1953 . The series will probably differ, however, from more recent groups in including a larger proportion of older children for whom surgical treatment did not become available until they were in their teens.

\section{Findings}

Of the 108 patients, $84(78 \%)$ were girls. Four cases were complicated by coarctation of the aorta of mild degree, unassociated with clinical evidence of the condition and insufficient to warrant resec- tion, one by a ventricular septal defect and one by congenital heart block.

The symptoms, physical signs, radiological and electrocardiographic findings are summarized in Table 1.

Symptoms. Excessive dyspnoea on exertion, undue fatigue, recurrent respiratory infections or failure to thrive were noted in 40 cases (37\%).

Physical Signs. Of the physical signs, the typical Gibson murmur was the most constant and best heard in the second left intercostal space, continuous throughout the cardiac cycle, with late systolic accentuation and a rattling 'machinery' quality. It was heard in $97(95 \%)$ of the patients.

In 11 cases the murmur was atypical. In four of these it was blowing rather than rattling. In three of the four the ductus was small with an external diameter of $4.5 \mathrm{~mm}$. or less and in the fourth the diameter was $9 \mathrm{~mm}$. In the remaining seven the diastolic element was soft and confined to early diastole. In these cases there was marked pulmonary hypertension, demonstrated by cardiac catheterization in five and at operation in two. This complication is known to cause a reduction in blood flow through the ductus and consequently an alteration in the character of the murmur.

A thrill was felt in 79 cases $(73 \%)$, continuous in $32(30 \%)$, systolic only in $47(43 \%)$.

The second heart sound in the pulmonary area is so loud in many children with normal hearts that it is often difficult to know if it is within the limits of normal or not. In the seven patients with pulmonary hypertension, however, it was definitely accentuated. In some cases the sound was obscured by the Gibson murmur. Splitting was usually heard, but with pulmonary hypertension it was occasionally single, due presumably to synchronous closure of the pulmonary and aortic valves.

A mid-diastolic murmur was heard in the mitral area in many of the cases, more frequently in those 
TABLE 1

SYMPTOMS, PHYSICAL SIGNS AND RADIOLOGICAL AND ELECTROCARDIOGRAPHIC FINDINGS

\begin{tabular}{|c|c|c|c|c|c|c|c|c|c|c|c|c|c|c|c|c|c|}
\hline \multirow[b]{3}{*}{ 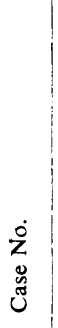 } & \multirow[b]{3}{*}{ 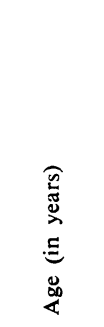 } & \multirow[b]{3}{*}{ 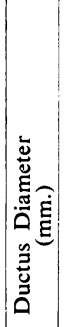 } & \multirow[b]{3}{*}{ 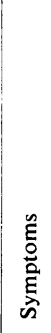 } & \multirow[b]{3}{*}{$\stackrel{\vec{\Xi}}{\underline{\Xi}}$} & \multirow[b]{3}{*}{ 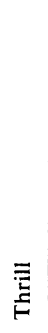 } & \multirow{3}{*}{ 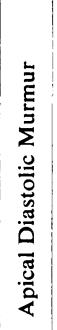 } & \multirow[b]{3}{*}{ 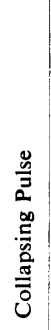 } & \multirow{2}{*}{\multicolumn{2}{|c|}{$\begin{array}{l}\text { Blood Pressure } \\
(\mathrm{mm} . \mathrm{Hg})\end{array}$}} & \multirow{2}{*}{\multicolumn{2}{|c|}{$\begin{array}{l}\text { Radiological } \\
\text { C/T Ratio }\end{array}$}} & \multicolumn{4}{|c|}{ Electrocardiogram } & \multirow[b]{3}{*}{ 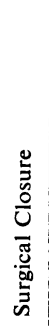 } & \multirow[b]{3}{*}{ 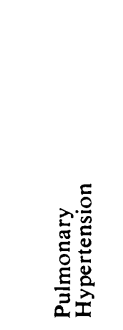 } \\
\hline & & & & & & & & & & & & \multicolumn{2}{|c|}{$\begin{array}{c}\text { L.V. } \\
\text { Dominance } \\
\mathbf{S v}_{1}+\mathbf{R v}_{6} \mathrm{~mm} . \\
\end{array}$} & \multicolumn{2}{|c|}{$\begin{array}{c}\text { R.V. } \\
\text { Dominance } \\
\text { R } \mathbf{v}_{1} \text { mm. }\end{array}$} & & \\
\hline & & & & & & & & 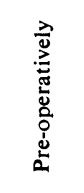 & 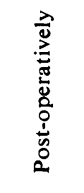 & 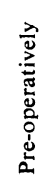 & 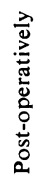 & 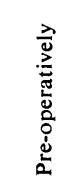 & 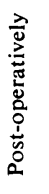 & 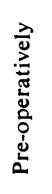 & 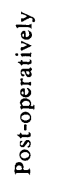 & & \\
\hline & & & & & & & & & $15 / 8$ & & & & & & & & \\
\hline & & & & & & $=$ & 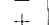 & $118 / 58$ & 120170 & 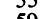 & 41 & si. & s. & & & & - \\
\hline 3 & $\begin{array}{ll}11 & 7 / 12\end{array}$ & W. & \pm & T. & C. & $\overline{-}$ & I & $\begin{array}{l}118 / 42 \\
\end{array}$ & $105 / 55$ & 47 & 48 & St. & St. & $\begin{array}{l}\text { St. } \\
\text { St. }\end{array}$ & $\begin{array}{l}\text { St. } \\
\text { St. }\end{array}$ & I. & - \\
\hline $\begin{array}{l}4 \\
5\end{array}$ & $\begin{array}{ll}5 & 7 / 12\end{array}$ & $?$ & + & $\mathrm{T}$. & C. & - & + & $120 / 50$ & $\begin{array}{l}120 / 70 \\
105 / 80\end{array}$ & 64 & 54 & St. & St. & St. & St. & I. & - \\
\hline $\begin{array}{l}3 \\
6 \\
7\end{array}$ & $\left|\begin{array}{rr}4 & 4 / 12 \\
14 & 3 / 12\end{array}\right|$ & \begin{tabular}{|l}
6 \\
$\mathbf{6}$ \\
$\mathbf{w}$
\end{tabular} & $\overline{\mathrm{s} .}$ & T. & S. & $=$ & $\bar{t}$ & $\begin{array}{r}84 / 48 \\
110 / 45\end{array}$ & $\begin{array}{l}105 / 80 \\
128 / 80\end{array}$ & 57 & $\begin{array}{l}51 \\
56\end{array}$ & $\begin{array}{l}\text { St. } \\
\text { St. }\end{array}$ & $\begin{array}{l}\text { St. } \\
\text { St. }\end{array}$ & $\begin{array}{l}\text { St. } \\
\text { St. }\end{array}$ & $\begin{array}{l}\text { St. } \\
\text { St. }\end{array}$ & $\begin{array}{l}\text { P. } \\
\text { I. }\end{array}$ & - \\
\hline $\begin{array}{l}7 \\
8 \\
0\end{array}$ & $\left|\begin{array}{cc}6 & 4 / 12 \\
5 & 10 / 12\end{array}\right|$ & $\begin{array}{l}8 \\
8\end{array}$ & $\begin{array}{l}+ \\
\text { D. }\end{array}$ & T. & $\begin{array}{l}\text { S. } \\
\text { S. }\end{array}$ & $=$ & $\frac{1}{-}$ & $\begin{array}{r}104 / 55 \\
84 / 44\end{array}$ & $\begin{array}{l}102 / 72 \\
110 / 78\end{array}$ & $\begin{array}{l}55 \\
50\end{array}$ & $\begin{array}{l}47 \\
53\end{array}$ & $\begin{array}{l}\text { St. } \\
\text { St. }\end{array}$ & $\begin{array}{l}\text { St. } \\
\text { St. }\end{array}$ & $\begin{array}{l}\text { St. } \\
\text { St. }\end{array}$ & $\begin{array}{l}\text { St. } \\
\text { St. }\end{array}$ & P. & $?+$ \\
\hline \begin{tabular}{r|}
9 \\
10
\end{tabular} & 7 & \begin{tabular}{|l}
8 \\
4
\end{tabular} & $=$ & $\mathrm{T}$. & C. & $=$ & - & $\begin{array}{r}74 / 34 \\
100 / 60\end{array}$ & $\begin{array}{r}82 / 48 \\
100 / 70\end{array}$ & St. & St. & St. & St. & St. & St. & P. & - \\
\hline 11 & 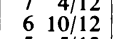 & $\begin{array}{l}4 \\
3\end{array}$ & - & $\begin{array}{l}\text { A. } \\
\text { A. }\end{array}$ & $\overrightarrow{\mathrm{s}}$. & 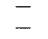 & $=$ & $\begin{array}{r}100 / 60 \\
93 / 38\end{array}$ & $\begin{array}{r}1007 / 70 \\
90 / 50\end{array}$ & $\begin{array}{l}50 \\
50\end{array}$ & $\begin{array}{l}46 \\
46\end{array}$ & $\overline{\text { St. }}$ & $\overline{\text { St. }}$ & $\overline{\text { St. }}$ & $\overline{\text { St. }}$ & $\begin{array}{l}\text { P. } \\
\text { P. }\end{array}$ & - \\
\hline $\begin{array}{l}12 \\
13\end{array}$ & $\left|\begin{array}{ll}5 & 5 / 12 \\
7 & 4 / 12\end{array}\right|$ & W. & \pm & T. & S. & $=$ & - & $\begin{array}{l}110 / 50 \\
118157\end{array}$ & $\begin{array}{l}115 / 80 \\
120 / 75\end{array}$ & $\begin{array}{l}55 \\
59\end{array}$ & $\begin{array}{l}41 \\
55\end{array}$ & $\begin{array}{l}\text { St. } \\
\text { St. }\end{array}$ & St. & $\begin{array}{l}\text { St. } \\
\text { St }\end{array}$ & St. & P. & - \\
\hline 14 & $\begin{array}{lll}1 & 4 & 4112 \\
6 & \end{array}$ & 6 & + & T. & C. & - & + & $\begin{array}{l}118 / 42 \\
118 / 2\end{array}$ & $\begin{array}{l}105 / 55 \\
1\end{array}$ & 47 & 48 & St. & St. & St. & St. & P. & $=$ \\
\hline $\begin{array}{l}15 \\
16\end{array}$ & $\begin{array}{|ll|}5 & 6 / 12 \\
8 & 5 / 12\end{array} \mid$ & $\begin{array}{l}4 \\
5\end{array}$ & \pm & T. & $\overline{s .}$ & $=$ & $\mp$ & $\begin{array}{r}102 / 60 \\
90 / 58\end{array}$ & $\begin{array}{l}110 / 80 \\
105 / 60\end{array}$ & $\begin{array}{l}54 \\
49\end{array}$ & $\begin{array}{l}52 \\
50\end{array}$ & $\begin{array}{l}\text { St. } \\
\text { St. }\end{array}$ & $\begin{array}{l}\text { St. } \\
\text { St. }\end{array}$ & $\begin{array}{l}\text { St. } \\
\text { St. }\end{array}$ & $\begin{array}{l}\text { St. } \\
\text { St. }\end{array}$ & $\begin{array}{l}\text { P. } \\
\text { P. }\end{array}$ & $\bar{z}$ \\
\hline $\begin{array}{l}17 \\
18\end{array}$ & $\left|\begin{array}{ll}6 & 4 / 12 \\
5 & 2 / 12\end{array}\right|$ & \begin{tabular}{|l|}
5 \\
6
\end{tabular} & \pm & T. & C. & - & \pm & $115 / 45$ & $\begin{array}{l}105 / 70 \\
115755\end{array}$ & $\begin{array}{l}54 \\
52\end{array}$ & $\begin{array}{l}51 \\
45\end{array}$ & $\begin{array}{l}\text { St. } \\
\text { St. }\end{array}$ & $\begin{array}{l}\text { St. } \\
\text { St. }\end{array}$ & $\begin{array}{l}\text { St. } \\
\text { St. }\end{array}$ & $\begin{array}{l}\text { St. } \\
\text { St }\end{array}$ & P. & 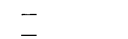 \\
\hline 19 & $\begin{array}{lll}12 & 10 / 12\end{array}$ & 10 & - & T. & s. & - & - & $\begin{array}{l}130 / 70 \\
130 / 70\end{array}$ & $\begin{array}{l}116 / 75 \\
115 / 60\end{array}$ & 46 & 44 & St. & St. & St. & St. & P. & - \\
\hline $\begin{array}{l}20 \\
21\end{array}$ & $\mid \begin{array}{ll}11 & 1 / 12 \\
10 & 3 / 12\end{array}$ & 5 & ${ }_{-}^{+}$ & $\mathrm{T}$. & C. & \pm & \pm & $\begin{array}{r}112400 \\
130 / 70\end{array}$ & $\begin{array}{l}11500 \\
110 / 70\end{array}$ & $\begin{array}{l}83 \\
49\end{array}$ & 55 & St. & St. & St. & St. & $\begin{array}{l}1 . \\
\text { p. }\end{array}$ & \pm \\
\hline $\begin{array}{l}22 \\
23\end{array}$ & $\left|\begin{array}{cc}7 & 5 / 12 \\
4 & 7 / 12\end{array}\right|$ & $\begin{array}{l}8 \\
6\end{array}$ & \pm & T. & $\overrightarrow{s .}$ & $\bar{t}$ & + & $\begin{array}{r}125 / 70 \\
90 / 45\end{array}$ & $\begin{array}{r}110 / 70 \\
90 / 60\end{array}$ & $\begin{array}{l}50 \\
55\end{array}$ & $\begin{array}{l}48 \\
52\end{array}$ & $\begin{array}{l}\text { St. } \\
45\end{array}$ & $\begin{array}{l}\text { St. } \\
29\end{array}$ & St. & St. & $\begin{array}{l}\text { P. } \\
\text { P. }\end{array}$ & $?-$ \\
\hline $\begin{array}{r}24 \\
25\end{array}$ & $\begin{array}{rr}6 & 8 / 1212 \\
12 & 2 / 12\end{array}$ & $\begin{array}{l}7 \\
9\end{array}$ & $\begin{array}{l}\text { D. } \\
+\end{array}$ & T. & S. & \pm & \pm & $\begin{array}{l}124 / 54 \\
104 / 60\end{array}$ & $11 \overline{5 / 80}$ & 54 & 49 & St. & St. & $\overline{\mathrm{St}}$ & St. & P. & - \\
\hline $\begin{array}{r}* 26 \\
27\end{array}$ & $\begin{array}{lll}4 & 10 / 12\end{array}$ & 9 & - & T. & S. & + & + & $\begin{array}{r}95 / 40 \\
\end{array}$ & $\begin{array}{l}105 / 70 \\
10165\end{array}$ & 64 & 58 & 57 & 55 & 6 & 5 & P. & - \\
\hline $\begin{array}{l}21 \\
28\end{array}$ & $\begin{array}{ll}4 & 8 / 12 \\
3 & 11 / 12\end{array}$ & . $\begin{array}{l}8 \\
5\end{array}$ & $\overline{-}$ & T. & s. & \pm & \pm & $\begin{array}{l}130 / 55 \\
104 / 70\end{array}$ & $\begin{array}{l}110 / 85 \\
115 / 80\end{array}$ & $\begin{array}{l}59 \\
59\end{array}$ & $\begin{array}{l}59 \\
51\end{array}$ & $\begin{array}{l}59 \\
31\end{array}$ & $\begin{array}{l}59 \\
27\end{array}$ & $\begin{array}{r}10 \\
6\end{array}$ & $\begin{array}{l}8 \\
7\end{array}$ & $\begin{array}{l}\text { R. } \\
\text { P. }\end{array}$ & $=$ \\
\hline $\begin{array}{l}29 \\
30\end{array}$ & $\begin{array}{ll}5 & 7 / 12 \\
9 & 5 / 12\end{array}$ & $\begin{array}{l}9 \\
7\end{array}$ & $=$ & $\mathrm{T}$. & S. & \pm & \pm & $\begin{array}{l}138 / 54 \\
114 / 78\end{array}$ & $\begin{array}{l}120 / 85 \\
105 / 80\end{array}$ & $\begin{array}{l}63 \\
53\end{array}$ & $\begin{array}{l}48 \\
45\end{array}$ & $\begin{array}{l}50 \\
56\end{array}$ & $\begin{array}{l}29 \\
36\end{array}$ & 8 & 4 & I. & $?+$ \\
\hline $\begin{array}{l}31 \\
32\end{array}$ & $\begin{array}{rr}6 & 10112\end{array}$ & 13 & + & A. & s. & + & + & $\begin{array}{l}115 / 185 \\
1150\end{array}$ & $\begin{array}{l}102 / 60 \\
105 / 160\end{array}$ & 64 & $\begin{array}{l}50 \\
50\end{array}$ & 86 & 50 & 15 & 9 & R. & + \\
\hline 33 & $\begin{array}{rr}10 & 9 / 12 \\
5 & 11 / 12\end{array}$ & $\begin{array}{r}12 \\
5\end{array}$ & \pm & T. & S. & $\begin{array}{l}+ \\
+\end{array}$ & $\begin{array}{l}+ \\
+\end{array}$ & $\begin{array}{l}130 / 40 \\
112 / 60\end{array}$ & $\begin{array}{l}115 / 60 \\
110 / 70\end{array}$ & $\begin{array}{l}53 \\
51\end{array}$ & $\begin{array}{l}43 \\
51\end{array}$ & 26 26 & $\begin{array}{l}36 \\
23\end{array}$ & $\begin{array}{l}16 \\
10\end{array}$ & $\begin{array}{l}16 \\
10\end{array}$ & P. & $\overline{-}$ \\
\hline $\begin{array}{l}34 \\
35\end{array}$ & $\begin{array}{cc}8 & 8 / 12 \\
5 & 512\end{array}$ & 15 & + & T. & $\begin{array}{l}\mathbf{S} . \\
\text {. }\end{array}$ & + & \pm & $\begin{array}{r}95 / 40 \\
110 / 35\end{array}$ & $\begin{array}{r}88 / 655 \\
11070\end{array}$ & $\begin{array}{l}64 \\
60\end{array}$ & $\begin{array}{l}56 \\
51\end{array}$ & $\begin{array}{l}74 \\
70 \\
50\end{array}$ & $\begin{array}{l}26 \\
32\end{array}$ & $\begin{array}{l}17 \\
22\end{array}$ & 22 & I. & $\bar{z}$ \\
\hline 36 & $14 \quad 3 / 12$ & 5 & - & $\mathrm{T}$. & - & - & + & $\begin{array}{l}120 / 65 \\
130175\end{array}$ & $\begin{array}{l}110 / 70 \\
110,70\end{array}$ & 51 & 46 & 47 & 37 & 6 & 7 & P. & - \\
\hline 38 & $\begin{array}{ll}10 & 2 / 12\end{array}$ & 6 & $\overline{\mathbf{D}}$. & $\mathbf{T}$. & $=$ & - & \pm & $125 / 66$ & $124 / 70$ & 46 & 47 & 52 & 33 & 9 & 7 & p. & $\overline{-}$ \\
\hline $\begin{array}{l}39 \\
40\end{array}$ & $\mid$\begin{tabular}{rr|r|r|r|}
4 & $111 / 12$ \\
10 & $9 / 12$
\end{tabular} & $\begin{array}{l}6 \\
7\end{array}$ & $\stackrel{+}{+}$ & T. & $=$ & $=$ & $\bar{t}$ & $\begin{array}{l}115 / 50 \\
100 / 50\end{array}$ & $\begin{array}{l}100 / 65 \\
120 / 70\end{array}$ & $\begin{array}{l}53 \\
56\end{array}$ & $\begin{array}{l}53 \\
50\end{array}$ & $\begin{array}{l}26 \\
32\end{array}$ & $\begin{array}{l}23 \\
20\end{array}$ & $\begin{array}{r}9 \\
10\end{array}$ & $\begin{array}{r}10 \\
5\end{array}$ & P. & $?+$ \\
\hline $\begin{array}{l}41 \\
42\end{array}$ & $\begin{array}{rr}3 & 3 / 12 \\
7\end{array}$ & $\begin{array}{l}9 \\
6\end{array}$ & D. & $T$. & C. & \pm & - & $\begin{array}{l}90 / 55 \\
125 / 15\end{array}$ & $\begin{array}{l}110 / 70 \\
11475\end{array}$ & 59 & $\begin{array}{l}55 \\
49 \\
\end{array}$ & 40 & 21 & 10 & 6 & P. & $?+$ \\
\hline 43 & $\begin{array}{ll}3 & 3 / 12 \\
& 3 / 12\end{array}$ & 6 & - & $\mathbf{T}$ & $\bar{c}$. & - & + & 5 & $98 / 62$ & 57 & 52 & 22 & 12 & 12 & 9 & P. & $?+$ \\
\hline $\begin{array}{l}44 \\
45\end{array}$ & $\begin{array}{ll}4 & 5 / 12\end{array}$ & $\begin{array}{l}8 \\
5\end{array}$ & $\bar{t}$ & T. & $\stackrel{+}{-}$ & \pm & \pm & $\begin{array}{l}100 / 45 \\
110 / 65\end{array}$ & $\begin{array}{l}102 / 60 \\
115 / 62\end{array}$ & & & $\begin{array}{l}44 \\
26\end{array}$ & $\begin{array}{l}37 \\
15 ?\end{array}$ & $\begin{array}{l}17 \\
10\end{array}$ & $\begin{array}{l}15 \\
5 ?\end{array}$ & $\begin{array}{l}\text { R. } \\
\text { P. }\end{array}$ & $?+$ \\
\hline $\begin{array}{l}46 \\
47\end{array}$ & $\begin{array}{rr}9 & 8 / 12 \\
11 & 9 / 12\end{array}$ & \begin{tabular}{r|r}
8 \\
11
\end{tabular} & - & $\mathrm{T}$. & $=$ & $=$ & \pm & $\begin{array}{r}125 / 65 \\
90 / 60\end{array}$ & $\begin{array}{l}120 / 80 \\
118755\end{array}$ & $\begin{array}{l}48 \\
50\end{array}$ & $\begin{array}{l}48 \\
49\end{array}$ & $\begin{array}{l}16 \\
40\end{array}$ & 14 & 3 & 2 & P. & $=$ \\
\hline 48 & $\begin{array}{ll}3 & 2 / 12 \\
3 & 2 / 12\end{array}$ & 7 & D. & $\mathrm{T}$. & s. & - & + & $112 / 60$ & $98 / 70$ & 56 & 51 & 33 & 33 & 10 & 13 & P. & $?+$ \\
\hline 50 & $\begin{array}{l}3 \\
2 \\
21 / 1 / 12\end{array}$ & 8 & $\overline{-}$ & T. & $\overline{s .}$ & $\begin{array}{l}- \\
-\end{array}$ & $\begin{array}{l}+ \\
+\end{array}$ & $\begin{array}{l}100 / 40 \\
110 / 50\end{array}$ & $\begin{array}{l}98 / 80 \\
110 / 85\end{array}$ & $\begin{array}{l}54 \\
58\end{array}$ & 52 & 52 & 35 & 18 & $\begin{array}{l}10 \\
15\end{array}$ & P. & \\
\hline $\begin{array}{l}51 \\
52\end{array}$ & 9/12 & $\begin{array}{r}15 \\
8\end{array}$ & \pm & A. & $s$ & $-\bar{t}$ & - & $\begin{array}{r}82 / 60 \\
130 / 70\end{array}$ & $10 \overline{5 / 65}$ & $\begin{array}{l}56 \\
60\end{array}$ & 54 & $\begin{array}{l}17 \\
33\end{array}$ & 23 & $\begin{array}{c}16 \\
7\end{array}$ & 5 & Ab. & $\pm_{-}^{+(100 / 70)}$ \\
\hline $\begin{array}{l}53 \\
54\end{array}$ & $8 / 12$ & 9 & D. & T. & s. & - & - & $\begin{array}{l}95 / 75 \\
95 / 40\end{array}$ & $110 / 60$ & 50 & 0 & 17 & 20 & 3 & 2 & P. & - \\
\hline 55 & $\begin{array}{ll}3 & 3 / 12 \\
3 & 3 / 12\end{array}$ & $\begin{array}{r}10 \\
6\end{array}$ & + & T. & s. & \pm & - & $\begin{array}{l}93 / 40 \\
110 / 75\end{array}$ & $10 \overline{2 / 70}$ & & & 30 & 28 & 22 & 20 & P. & \\
\hline $\begin{array}{l}56 \\
57\end{array}$ & $\begin{array}{ll}3 & 22 / 12 \\
5 & 7 / 12\end{array}$ & $\begin{array}{l}7 \\
6\end{array}$ & D. & T. & $\stackrel{+}{\text { s. }}$ & \pm & $\stackrel{+}{+}$ & $\begin{array}{l}108 / / 50 \\
100 / 75\end{array}$ & $\begin{array}{r}115 / 70 \\
98 / 72\end{array}$ & $\begin{array}{l}58 \\
51\end{array}$ & $\begin{array}{l}54 \\
51\end{array}$ & $\begin{array}{l}45 \\
37\end{array}$ & 23 & $\begin{array}{l}9 \\
9\end{array}$ & $\begin{array}{l}10 \\
8\end{array}$ & $\begin{array}{l}\mathbf{P} . \\
\mathbf{P}\end{array}$ & $=$ \\
\hline $\begin{array}{l}58 \\
59\end{array}$ & \begin{tabular}{ll|}
6 & $4 / 12$ \\
6 & $5 / 12$
\end{tabular} \mid & 8 & + & $\mathrm{T}$. & C. & \pm & $=$ & $\begin{array}{l}90 / 55 \\
110 / 60\end{array}$ & $\begin{array}{r}94 / 60 \\
116 / 68\end{array}$ & $\frac{5}{57}$ & 56 & 23 & 21 & 3 & $\begin{array}{l}3 \\
7\end{array}$ & P. & - \\
\hline 60 & $\begin{array}{lll}5 & 7 / 12\end{array}$ & $\begin{array}{l}0 \\
? \\
?\end{array}$ & + & $\mathrm{T}$. & + & $=$ & + & $\begin{array}{l}1101 / 60 \\
135 / 68\end{array}$ & $\begin{array}{l}11101 / 86 \\
12 / 86\end{array}$ & 60 & 49 & 43 & 30 & 8 & 5 & P. & - \\
\hline $\begin{array}{l}61 \\
62\end{array}$ & $\begin{array}{ll}9 & 1 / 12 \\
2 & 2 / 12\end{array}$ & $\begin{array}{r}11 \\
8\end{array}$ & \pm & T. & S. & \pm & $\begin{array}{l}+ \\
+\end{array}$ & $\begin{array}{r}115 / 55 \\
90 / 40\end{array}$ & $\begin{array}{l}1168 / 80 \\
100 / 70\end{array}$ & $\begin{array}{l}59 \\
60\end{array}$ & $\begin{array}{l}54 \\
54\end{array}$ & $\begin{array}{l}49 \\
41\end{array}$ & $\begin{array}{l}33 \\
20\end{array}$ & $\begin{array}{r}10 \\
9\end{array}$ & $\begin{array}{c}10 \\
8\end{array}$ & $\begin{array}{l}\text { R. } \\
\text { P. }\end{array}$ & - \\
\hline $\begin{array}{l}63 \\
64\end{array}$ & $9 / 12$ & 5 & + & A. & $\vec{s}$ & $\bar{t}$ & + & $\begin{array}{l}98 / 55 \\
95 / 55\end{array}$ & $\begin{array}{r}93 / 60 \\
114 / 70\end{array}$ & $\begin{array}{l}59 \\
55\end{array}$ & $\begin{array}{l}49 \\
49\end{array}$ & $\begin{array}{l}26 \\
50\end{array}$ & 22 & 10 & 10 & P. & $=$ \\
\hline 65 & $109 / 12$ & 8 & D. & $\mathrm{T}$. & C. & +1 & - & $100 / 70$ & $98 / 80$ & 51 & 48 & 43 & (1) & 6 & 5 & P. & - \\
\hline 67 & $\begin{array}{lll}4 & 10 / 12\end{array}$ & $\begin{array}{l}6 \\
8\end{array}$ & D. & T. & - & 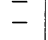 & + & $\begin{array}{l}120 / 65 \\
105 / 60\end{array}$ & $\begin{array}{l}110080 \\
100 / 70\end{array}$ & $\begin{array}{l}47 \\
53\end{array}$ & $\begin{array}{l}41 \\
51\end{array}$ & 26 & 19 & 5 & 3 & P. & $\bar{z}$ \\
\hline 70 & & 8 & & & & & & & $98 / 60$ & 52 & & 14 & & 10 & 10 & & \\
\hline
\end{tabular}


TABLE 1-continued

\begin{tabular}{|c|c|c|c|c|c|c|c|c|c|c|c|c|c|c|c|c|c|}
\hline \multirow[b]{3}{*}{$\begin{array}{l}\dot{0} \\
z \\
\ddot{v} \\
\tilde{U}\end{array}$} & \multirow[b]{3}{*}{ 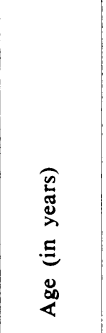 } & \multirow[b]{3}{*}{ 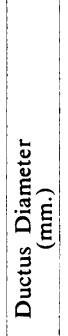 } & \multirow[b]{3}{*}{ 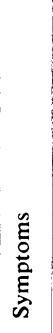 } & \multirow[b]{3}{*}{ 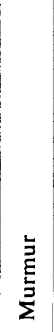 } & \multirow[b]{3}{*}{ 豆 } & \multirow{3}{*}{ 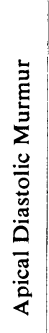 } & \multirow[b]{3}{*}{ 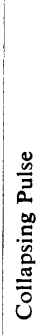 } & \multirow{2}{*}{\multicolumn{2}{|c|}{$\begin{array}{l}\text { Blood Pressure } \\
\text { (mm. Hg.) }\end{array}$}} & \multirow{2}{*}{\multicolumn{2}{|c|}{$\begin{array}{c}\text { Radiological } \\
\text { C/T Ratio }\end{array}$}} & \multicolumn{4}{|c|}{ Electrocardiogram } & \multirow[b]{3}{*}{ 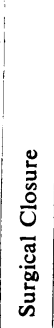 } & \multirow[b]{3}{*}{ 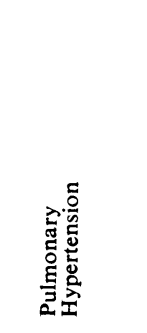 } \\
\hline & & & & & & & & & & & & \multicolumn{2}{|c|}{$\begin{array}{c}\text { L.V. } \\
\text { Dominance } \\
\mathbf{S v}_{1}+\mathbf{R v}_{6} \mathrm{~mm} .\end{array}$} & \multicolumn{2}{|c|}{$\begin{array}{l}\text { R.V. } \\
\text { Dominance } \\
\text { Rv }_{1} \mathrm{~mm} \text {. }\end{array}$} & & \\
\hline & & & & & & & & 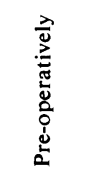 & 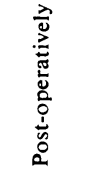 & 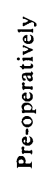 & 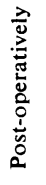 & 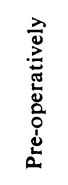 & 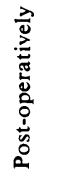 & 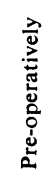 & 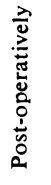 & & \\
\hline & $911 / 12$ & & D. & $\mathrm{T}$. & C. & - & $t$ & $120 / 50$ & $106 / 68$ & 46 & 44 & 46 & 36 & 6 & 5 & P. & - \\
\hline 72 & $\begin{array}{ll}3 & 1112 \\
3 & 3 / 12\end{array}$ & $\begin{array}{l}0 \\
9\end{array}$ & D. & $\mathrm{T}$. & C. & $\vec{t}$ & + & $115 / 38$ & $115 / 75$ & 63 & 55 & 30 & 18 & 6 & 5 & P. & - \\
\hline 73 & \begin{tabular}{rr|}
7 & $10 / 12$
\end{tabular} \mid & 8 & - & $\mathrm{T}$. & C. & + & - & $100 / 65$ & $116 / 72$ & 50 & 50 & 41 & 33 & 6 & 5 & $P$. & $\overline{-}$ \\
\hline 74 & $\begin{array}{ll}3 & 5 / 12\end{array}$ & 16 & + & A. & s. & + & - & $90 / 50$ & $100 / 55$ & 62 & 59 & 38 & - & 8 & - & 1. & + \\
\hline 75 & $5 \quad 4 / 12$ & 7 & D. & T. & C. & - & - & $110 / 75$ & $100 / 65$ & 53 & 55 & 50 & 39 & 10 & 8 & P. & - \\
\hline 76 & $210 / 12$ & 9 & - & T. & C. & + & - & $110 / 50$ & $90 / 6$ & 62 & 59 & 65 & 45 & 10 & 10 & P. & - \\
\hline $76 \mathrm{~A}$ & $55 / 12$ & 6 & - & $\mathrm{T}$. & s. & - & + & $100 / 35$ & $94 / 54$ & 54 & 52 & 39 & 39 & 4 & 5 & P. & - \\
\hline $65 \mathrm{~B}$ & $1 \quad 1 / 12$ & 8 & + & $\mathrm{T}$. & c. & t & - & $145 / 55$ & - & 61 & 51 & 41 & - & 14 & - & P. & - \\
\hline 77 & $5 \quad 8 / 12$ & 6 & + & $\mathbf{T}$. & C. & + & + & $110 / 5$ & $105 / 65$ & 50 & 48 & 18 & 15 & 4 & 5 & P. & - \\
\hline $\begin{array}{l}78 \\
79\end{array}$ & $29 / 12$ & 8 & D. & T. & S. & - & - & $100 / 60$ & $105 / 60$ & 57 & 54 & 21 & 20 & 4 & 6 & P. & - \\
\hline $\begin{array}{l}79 \\
81\end{array}$ & $\begin{array}{rr}9 & 11 / 12 \\
14 & 1 / 12\end{array}$ & 7 & \pm & $\mathbf{T}$. & C. & $\bar{z}$ & $\bar{z}$ & $\begin{array}{l}140 / 75 \\
150 / 70\end{array}$ & $\begin{array}{l}138 / 88 \\
130 / 80\end{array}$ & $\begin{array}{l}47 \\
57\end{array}$ & $\begin{array}{l}4 \\
4\end{array}$ & 38 & $\begin{array}{l}27 \\
32\end{array}$ & 3 & 2 & $P$. & 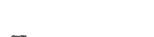 \\
\hline $\begin{array}{r}81 \\
.82\end{array}$ & $\left|\begin{array}{rr}14 & 1 / 12 \\
5 & 10 / 12\end{array}\right|$ & r & $\overline{\mathrm{D}}$. & T. & C. & $\overline{-}$ & - & $92 / 56$ & $98 / 68$ & 55 & 55 & $\begin{array}{l}40 \\
24\end{array}$ & $\begin{array}{l}32 \\
20\end{array}$ & $\begin{array}{r}1 \\
10\end{array}$ & $\begin{array}{l}1 \\
7\end{array}$ & P. & - \\
\hline 83 & $\begin{array}{ll}13 & 6 / 12\end{array}$ & $i$ & D. & T. & s. & - & - & $125 / 75$ & $120 / 80$ & 49 & 46 & 25 & 20 & 2 & - & P. & - \\
\hline$* 84$ & $\begin{array}{lll}3 & 11 / 12\end{array}$ & 10 & - & T. & + & - & + & $125 / 45$ & $118 / 80$ & 67 & 55 & 52 & 25 & 4 & 8 & $P$. & - \\
\hline 85 & $135 / 12$ & 11 & + & T. & S. & + & + & $94 / 48$ & $115 / 85$ & 54 & 44 & 46 & 40 & 1 & 1 & P. & - \\
\hline 86 & $58 / 12$ & 4 & - & T. & S. & - & + & $102 / 64$ & 112 & 56 & 50 & 29 & 28 & 2 & 2 & P. & - \\
\hline $\begin{array}{l}87 \\
88\end{array}$ & $58 / 12$ & 7 & D. & $\mathrm{T}$. & S. & - & + & $102 / 50$ & $104 / 60$ & 54 & 5 & 46 & 33 & 3 & 3 & $P$. & $-(100 / 60)$ \\
\hline $\begin{array}{l}88 \\
89\end{array}$ & $\begin{array}{ll}7 & \\
4 & 2 / 12\end{array}$ & $\begin{array}{r}10 \\
6\end{array}$ & \pm & $\mathrm{T}$. & S. & \pm & \pm & $\begin{array}{l}105 / 85 \\
117 / 60\end{array}$ & $\begin{array}{l}110 / 85 \\
110 / 82\end{array}$ & $\begin{array}{l}63 \\
54\end{array}$ & $\overline{58}$ & $\begin{array}{l}52 \\
26\end{array}$ & 30 & $\begin{array}{l}5 \\
6\end{array}$ & $\overline{12}$ & P. & $\pm(100 / 60)$ \\
\hline 90 & $4 \quad 6 / 12$ & 6 & - & T. & s. & - & - & $115 / 65$ & 110 & 59 & 56 & 32 & 28 & 4 & 5 & P. & 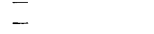 \\
\hline $90_{\mathrm{A}}$ & $95 / 12$ & 7 & - & T. & S. & + & + & $125 / 64$ & & 51 & 49 & 3 & 32 & 5 & 5 & P. & - \\
\hline 91 & $3 \quad 4 / 12$ & 5 & D. & T. & - & - & + & $110 / 64$ & $100 /$ & 51 & 56 & 2 & & 7 & - & P. & - \\
\hline 94 & $2 \quad 1 / 12$ & 14 & + & A. & S. & + & - & $80 / ?$ & $90 / 6$ & 65 & 6 & 41 & 27 & 13 & 13 & I. & - \\
\hline $\begin{array}{l}95 \\
96\end{array}$ & $511 / 12$ & 5 & - & $\mathrm{T}$. & - & - & + & 120 & 120 & 57 & 5 & 26 & $\overline{30}$ & 8 & 7 & P. & - \\
\hline $\begin{array}{l}96 \\
97\end{array}$ & $\begin{array}{ll}6 & 1 / 12 \\
7 & 6 / 12\end{array}$ & $\begin{array}{l}5 \\
5\end{array}$ & $\bar{\perp}$ & $\mathrm{T}$. & $\overline{\mathbf{S}}$ & $\overrightarrow{+}$ & $=$ & $\begin{array}{l}110 \\
120\end{array}$ & $\begin{array}{r}118 / 80 \\
88 / 70\end{array}$ & $\begin{array}{l}49 \\
42\end{array}$ & $\begin{array}{l}51 \\
43\end{array}$ & $\begin{array}{l}29 \\
24\end{array}$ & 29 & $\begin{array}{r}2 \\
10\end{array}$ & $\underline{3}$ & P. & $\overline{-}$ \\
\hline 98 & $\begin{array}{ll}7 & 6 / 12 \\
5 & 6 / 12\end{array}$ & 8 & + & $\mathrm{T}$ & S. & + & + & $115 / 50$ & $120 / 8$ & 61 & 60 & 45 & - & 8 & 二 & P. & $\bar{z}$ \\
\hline 99 & $\begin{array}{ll}6 & 6 / 12\end{array}$ & 16 & - & A. & s. & + & - & $115 /$ & - & 59 & - & 36 & - & 7 & - & Ab. & $+(64$ mean $)$ \\
\hline 101 & 4 & 10 & + & A. & S. & + & + & $115 / 55$ & $110 / 80$ & 67 & 60 & 22 & - & 9 & - & P. & $+(82 / 47)$ \\
\hline 102 & $1 \quad 11 / 12$ & 14 & + & A. & S. & + & + & 115 & $110 / 70$ & 70 & 63 & 26 & 26 & 5 & 5 & 1. & $+(76 / 42)$ \\
\hline 103 & $13 / 12$ & 7 & D. & T. & S. & - & - & $110 / 55$ & 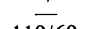 & 58 & $5 \varepsilon$ & $\mathbf{S}$ & St. & St. & St. & $P$. & - \\
\hline 104 & $\begin{array}{lll}5 & 10 / 12\end{array}$ & 9 & D. & T. & - & - & - & $93 / 50$ & $110 / 60$ & 57 & 48 & 20 & - & 1 & - & P. & - \\
\hline 106 & $5 \quad 6 / 12$ & 7 & + & $\mathrm{T}$. & - & - & $\ldots$ & $85 / 60$ & $110 / 80$ & 59 & 51 & 2. & - & 8 & - & P. & - \\
\hline 107 & $411 / 12$ & 7 & - & $\mathrm{T}$. & C. & + & -- & $116 / 68$ & $110 / 70$ & 54 & 54 & 37 & - & 2 & - & P. & - \\
\hline 108 & $510 / 12$ & 7 & - & T. & C. & + & - & $116 / 58$ & $120 / 65$ & 61 & 60 & 33 & 30 & 2 & 2 & P. & - \\
\hline$* 23 / 1$ & $119 / 12$ & 10 & - & T. & C. & - & - & $105 / 45$ & $115 / 85$ & 60 & 57 & St. & St. & St. & St. & P. & + \\
\hline$* 23 / 2$ & $4 \quad 2 / 12$ & 6 & + & T. & - & + & - & $105 / 70$ & $95 / 65$ & 54 & 55 & 43 & 28 & 9 & 5 & P. & - \\
\hline$* 23 / 3$ & $29 / 12$ & 10 & - & T. & S. & + & + & 120 & 102 & 58 & 53 & 4 & 27 & 15 & 15 & P. & + \\
\hline${ }^{*} 23 / 4$ & $211 / 12$ & 10 & - & T. & C. & - & & $105 / 55$ & $110 / 82$ & 61 & - & 42 & St. & 2 & St. & P. & $\ldots$ \\
\hline
\end{tabular}

$\mathrm{T}=$ typical machinery murmur, $\mathbf{A} .=$ atypical murmur, $\mathrm{C} .=$ continuous, $\mathrm{S} .=$ systolic, $\mathrm{St} .=$ standard leads $1,2,3, \mathbf{P} .=$ permanent, $\mathbf{R} .=$ recurrence I. $=$ incomplete, $A$ b. $=$ abandoned, $\mathrm{D} .=$ doubtful, being retrospective, $\mathrm{W} .=$ wide.

* Associated abnormality: 26, congenital heart block; 84, ventricular septal defect; 23/1, 23/2, 23/3, 23/4, slight coarctation of aorta.

seen later in the series. It was recognized only once in the first 20 patients but was heard in 53 of the remaining 88 , and this latter proportion probably represents its true incidence.

The pulse was described as 'collapsing' in 53 cases $(49 \%)$. In many normal children the diastolic fall of pressure is rapid and the pulse more collapsing than in an adult. It is therefore in some cases difficult to decide if it is abnormally collapsing or not. One would expect this difficulty to be less when the pulse pressure is large, and Table 2 demonstrates that the higher the pulse pressure the larger was the percentage of pulses recorded as collapsing.
TABLE 2

PULSE PRESSURES RELATED TO COLLAPSING PULSE

\begin{tabular}{|c|c|c|c|}
\hline Pulse & Pressure $(\mathrm{mm} . \mathrm{Hg})$ & Collapsing Pulse & Total \\
\hline & $\begin{array}{ll}40 \text { or less } & . \\
41-50 & . \\
51-60 & . \\
61-70 & . \\
71 \text { and over }\end{array}$ & $\begin{array}{r}7(23 \%) \\
11(46 \%) \\
16(53 \%) \\
8(80 \%) \\
9(75 \%)\end{array}$ & $\begin{array}{l}30 \\
24 \\
30 \\
10 \\
12\end{array}$ \\
\hline & & $51(49 \%)$ & 106 \\
\hline
\end{tabular}

Two cases are omitted as the blood pressure readings before operation were unsatisfactory.

Radiographs. For the analysis of the radiological findings we are indebted to Dr. J. K. Walker. He 
first estimated the size of the heart by means of the cardio-thoracic ratio. Taking the upper limit of normal to be $52 \%$, he found evidence of cardiac enlargement in $70 \%$ of cases. Although this ratio is not a completely satisfactory measure of the actual size of the heart it does offer a simple and reasonably accurate method of comparing heart size at different times in the same individual, and it will be seen from Table 3 that the cardio-thoracic ratio tended to increase with the diameter of the ductus found at operation.

TABLE 3

RELATION OF CARDIO-THORACIC RATIO TO SIZE OF PATENT DUCTUS

\begin{tabular}{|c|c|c|c|}
\hline $\begin{array}{c}\text { Cardio-thoracic } \\
\text { Ratio }(\%)\end{array}$ & $\begin{array}{l}\text { Patent Ductus } \\
\text { Arteriosus up to } \\
7 \mathrm{~mm} \text {. Diameter }\end{array}$ & $\begin{array}{c}\text { Diameter } \\
7 \cdot 5-10 \mathrm{~mm} .\end{array}$ & $\begin{array}{c}\text { Diameter } \\
10 \text { mm. and } \\
\text { Over }\end{array}$ \\
\hline $\begin{array}{l}52 \text { or less } \\
53-56 \quad \ldots \\
57-60 \quad \ldots \\
61-64 \text {. } \\
65 \text { or over }\end{array}$ & $\begin{array}{c}22(46 \%) \\
16(33 \%) \\
8(16 \%) \\
3(6 \%) \\
-\end{array}$ & $\begin{aligned} 7 & (21 \%) \\
4 & (12 \%) \\
13 & (40 \%) \\
7 & (21 \%) \\
2 & (6 \%)\end{aligned}$ & $\begin{array}{l}1(7 \%) \\
3(21 \%) \\
4(28 \%) \\
3(21 \%) \\
2(14 \%)\end{array}$ \\
\hline Total & 49 & 33 & 13 \\
\hline
\end{tabular}

He then applied Meyer's more accurate method of diagnosing enlargement of the heart, in which the cardiac area is calculated from the long and broad diameters of the heart in the postero-anterior film and compared with the cardiac area of a normal child of similar height and weight (Meyer, 1949). By this means, cardiac enlargement was suggested in $74 \%$ of the patients.

It is possible that if we had used Lind's method of calculating the cardiac volume we would have found that there was some increase in volume in a still higher proportion of cases (Lind, 1950). The left ventricle appeared to be enlarged in 86 cases $(81 \%)$, the pulmonary arc unduly prominent in $93(87 \%)$, the hilar vessels enlarged in $95(89 \%)$ and the lung fields hyperaemic in $97(91 \%)$. Expansile pulsation of the hilar vessels was detected in 27 cases $(25 \%)$.

Electrocardiographs. All but two patients were electrocardiographed. In 23 of the early cases the standard leads only were employed but in the other 83, aVR, aVL, aVF, $\mathrm{V}_{1}, \mathrm{~V}_{3}, \mathrm{~V}_{6}$ and latterly $\mathrm{V}_{3 \mathrm{R}}$ were also recorded. The electrocardiograph (E.C.G.) was normal in 42 of the 83 cases. A considerable number, however, showed changes which are regarded by some authors (Sodi-Pallares and Marsico, 1955; Landtman, 1954) as indicative of left ventricular dilatation, namely, excessive amplitude of $R_{\mathrm{v} 6}$ and $S_{\mathrm{v} 1}$, the sum of the two exceeding $35 \mathrm{~mm}$., together with a tall upright $\mathrm{T}_{\mathrm{v} 6}$. In 33 of the 83 patients $(40 \%)$, the above criteria of left ventricular dilatation were found. In a further two, excessively tall $R$ waves with inverted $T$ waves in lead $V_{6}$ suggested left ventricular hypertrophy rather than dilatation (Landtman, 1954).

Evidence of right ventricular hypertrophy, namely, $\mathbf{R}_{\mathrm{v} 1}$ greater than $10 \mathrm{~mm}$. (Switzer and Besoain, 1950), was found in 14 cases. In four it occurred without evidence of left ventricular hypertrophy or dilatation. In two of these, a pulmonary artery pressure almost at systemic level suggested that the shunt might soon have become reversed, and in one such reversal was in fact seen under the conditions of thoracotomy. In the other two, with $R_{V_{1}}$ equalling 10 and $13 \mathrm{~mm}$. respectively, significant pulmonary hypertension was not noted at operation, and the ductus, $6 \mathrm{~mm}$. in diameter in each, was of a size not usually accompanied by this complication. In an additional case without pulmonary hypertension, $R_{\mathrm{V} 1}$ of $10 \mathrm{~mm}$. and $\mathrm{S}_{\mathrm{v} 6}$ of $14 \mathrm{~mm}$. also satisfied Orme's criteria for right ventricular hypertrophy (Orme and Adams, 1952). In the remaining 10 cases evidence of right ventricular hypertrophy was accompanied by that of left ventricular hypertrophy or dilatation. In only seven of these was there definite evidence of pulmonary hypertension at operation or on cardiac catheterization. In the other three, with $R_{\mathrm{V} 1} 20,15$ and $14 \mathrm{~mm}$. respectively, there was no obvious pulmonary hypertension and therefore possibly no right ventricular hypertrophy. On the other hand, the ductus was of moderate size, $8 \mathrm{~mm}$. in diameter, in two of them, and large, $14 \mathrm{~mm}$. in diameter, in the other, and it seems perhaps more probable that there was in all three a degree of pulmonary hypertension sufficient to have caused some right ventricular hypertrophy but not to have resulted in gross macroscopic changes in pulmonary vasculature by the time the operation was performed.

Cardiac Catheterization. Cardiac catheterization was only performed, first, in those cases in which there was any doubt about the presence of a patent ductus arteriosus after the physical signs and radiological and E.C.G. findings had been considered, or, secondly, when significant pulmonary hypertension was thought to be present. It was undertaken, therefore, when the murmur was atypical or very loud and accompanied by an unusually loud second sound, and sometimes also when radiographs showed marked hilar enlargement and a very large heart. It was, however, not available for many of the early patients whom we might have wished to investigate in this way and was employed in only seven. In all these, patency of the ductus was indicated by the demonstration of increased oxygenation of the blood in the 
pulmonary artery and in three it was confirmed by passing the catheter through it into the aorta. Significant pulmonary hypertension was recorded in six; in the seventh the record was unsatisfactory but also suggested pulmonary hypertension. In one additional cyanosed patient the ductus was catheterized and the oxygen saturation of the blood from the aorta was shown to be reduced, indicating a rightto-left shunt from pulmonary artery to aorta. The child was, therefore, considered to be unsuitable for surgery and was excluded from this series.

Surgical Procedure. In each of the 108 patients thoracotomy was performed under anaesthesia with intravenous barbiturates, relaxants and nitrous oxide, and the diagnosis was confirmed. In 107, closure of the ductus was attempted. In 103 of these cases, double ligation with strong linen thread appeared to abolish the flow through the ductus. In three simple ligation was impossible owing to the great width of the vessel, 14-16 mm. diameter, and to the very high pressure within it. Partial closure was, however, effected by a pad tied firmly over the vessel which definitely reduced the shunt and resulted in an immediate diminution in heart size and a significant rise of diastolic pressure. Ligation of a high-pressure ductus was facilitated in some cases by the induction of hypotension by a ganglionblocking agent such as 'arfonad'. In another patient brisk haemorrhage occurred during the dissection of a high-pressure sclerotic ductus and the operation was abandoned. There were no ill effects from this operation and the case is still under review. In the remaining case the shunt, which had been left to right during cardiac catheterization, was found to be reversed under the conditions of the operation and closure was not attempted. The patient died three days later. Necropsy was unfortunately not performed.

\section{Results of Operation}

Symptoms. In each of the 40 children who presented with symptoms operation was followed by definite improvement, and in a further $20(18 \%)$ follow-up revealed that pre-operative limitation of activity had passed unnoticed by the patient's family, as increase in the child's exercise tolerance was noted by parents who had mistakenly considered his energy to have been normal before operation.

Physical Signs. These were considered as murmur and blood pressure readings.

MURmur. In 92 of the 108 patients $(85 \%)$ the diagnostic murmur was no longer audible after operation and did not recur during the follow-up period of at least six months. In many of these cases a systolic murmur persisted in the pulmonary area. It was usually soft and varied in intensity with respiration and was considered to be of no significance.

In seven patients, all with a patent ductus of $9 \mathrm{~mm}$. or more in diameter, a continuous murmur was heard within two weeks of operation despite apparently satisfactory ligation. As dressings were not removed during the first few days to facilitate auscultation, it is not known if in some of these cases the murmur was audible immediately after the operation: in others it definitely did not develop until during the second week. In all seven, however, the diastolic blood pressure rose after operation by at least $15 \mathrm{~mm}$. $\mathrm{Hg}$ and there was radiological evidence of a decrease in the size of the heart. In two of these cases the murmur ceased after about a year, indicating spontaneous closure, which may therefore still take place in the others. In four cases the Gibson murmur recurred one to 12 months after operation. In two of these the ductus has been retied and the murmur has ceased. The third refused a second operation and the fourth is still being considered for further surgery. In the three cases in which a pad was used to compress the ductus only partial closure was achieved and the continuous murmur persisted. In the remaining two patients, the one with the friable ductus and the other with the reversed shunt at operation, ligation was not performed.

Blood Pressure. The immediate effect of ligation of the ductus was a rise in the systolic and diastolic blood pressure. The systolic pressure fell again over the course of a week or so but the diastolic remained higher than the pre-operative level, being over $50 \mathrm{~mm}$. $\mathrm{Hg}$ in all but two patients, whose pressure was $48 \mathrm{~mm}$. $\mathrm{Hg}$. The distribution of the diastolic blood pressures before and after operation is set out in Table 4.

TABLE 4

DIASTOLIC BLOOD PRESSURE BEFORE AND AFTER OPERATION

\begin{tabular}{lll|c|c}
\hline \multicolumn{2}{l|}{ Pressure (mm. Hg) } & Pre-operative & Post-operative \\
\hline 30 or less & $\ldots$ & $\ldots$ & $2(2 \%)$ & - \\
$31-40$ & $\ldots$ & $\ldots$ & $13(13 \%)$ & $22(2 \%)$ \\
$41-50$ & $\ldots$ & $\ldots$ & $22(22 \%)$ & $19(19 \%)$ \\
$51-60$ & $\ldots$ & $\ldots$ & $30(30 \%)$ & $79(79 \%)$ \\
61 and over &. & $33(33 \%)$ & 100 \\
\hline
\end{tabular}
(Cases $17,24,43,51,76 B, 88,99,103$ are omitted owing to inadequate
data.)

Radiographs. The rise in the diastolic level of the systemic blood pressure with the abolition of the 
aorta-pulmonary shunt might be expected to be paralleled by a reduction in the size of the heart. This was seen on $x$-ray examination in 79 patients $(64 \%)$, including many in whom the radiological appearances before operation were considered to be within normal limits. The comparison between the pre-operative and post-operative radiographs could not be made in a certain number of cases for various reasons, such as incomplete follow-up, difference in positioning for radiography, etc. A film at an interval of three months was usually used for the post-operative study and any reduction in the cardio-thoracic ratio can therefore be assumed to be independent of general growth of the body relative to the heart. A reduction in the cardiothoracic ratio at this interval has been interpreted as an indication of absolute reduction in the size of the heart. In Table 5 the distribution of the percentage reduction in the cardio-thoracic ratio is set out. The table only includes those cases in which the external diameter of the ductus was recorded and in which comparable pre-operative and post-operative radiographs were available, and it is excessively weighted with patients with small ductuses. It does, however, tend to confirm that ligation of a large ductus often leads to a considerable reduction in heart size. In films taken 18 months or longer after operation this reduction was in many cases even more marked.
The $T$ wave became smaller in $V_{6}$ in all but six cases, confirming a decrease in left ventricular dilatation, and upright in the two cases in which it was inverted before operation, suggesting a decrease in left ventricular hypertrophy. In the 14 patients whose E.C.G. records suggested right ventricular hypertrophy, the post-operative findings were as follows. In the nine patients with recognized pulmonary hypertension the E.C.G. was not available in one case owing to post-operative death; in three, the evidence of right ventricular hypertrophy disappeared, and in the remaining five it persisted. Of the five patients not thought to have had pulmonary hypertension no post-operative record was available in one, and $V_{1}$ became normal after operation in only one. In the additional case without pulmonary hypertension, in which $\mathbf{R}_{\mathrm{v} 1}$ was $10 \mathrm{~mm}$. and $S_{\mathrm{v} 6} 14 \mathrm{~mm}$., the E.C.G. showed no change following operation.

\section{Discussion}

Aetiology. The physical phenomenon of spontaneous closure of the ductus arteriosus is not understood. The fundamental cause of persistent patency of the ductus is therefore also unknown. In this series there is the usual marked predominance of females, which may suggest an endocrine factor. There is only one instance of maternal rubella during pregnancy, a lower incidence than in some

TABLE 5

POST-OPERATIVE REDUCTION IN CARDIO-THORACIC RATIO

\begin{tabular}{|c|c|c|c|c|c|}
\hline Ductus Diameter (mm.) & $\begin{array}{l}\text { Cardio-thoracic Decrease } \\
\text { Less than } 4 \%\end{array}$ & $\begin{array}{c}4-7 \% \\
\text { Decrease }\end{array}$ & $\begin{array}{l}8-11 \% \\
\text { Decrease }\end{array}$ & $\begin{array}{l}12 \% \text { Decrease } \\
\text { and Over }\end{array}$ & Total \\
\hline $\begin{array}{l}4 \text { or less } \\
7 \cdot 5-10 \\
10 \cdot 5 \text { and over }\end{array}$ & $\begin{array}{r}31 \\
16 \\
5\end{array}$ & $\begin{array}{r}4 \\
11 \\
2\end{array}$ & $\overline{7}$ & $\begin{array}{r}- \\
2\end{array}$ & $\begin{array}{l}35 \\
35 \\
12\end{array}$ \\
\hline & 52 & 17 & 10 & 3 & 82 \\
\hline
\end{tabular}

Electrocardiographs. A similar trend was seen in the E.C.G. records of the 35 cases showing left ventricular dilatation or hypertrophy before operation. Thirty had comparable post-operative records of which 24 were within normal limits, the sum of $S_{v 1}$ and $R_{v 6}$ being less than $35 \mathrm{~mm}$., suggesting a decrease in the size of the left ventricle. One of the six, with a persistently abnormal E.C.G., had an associated congenital heart block and the persistent enlargement of the left ventricle may be due to the high stroke output necessitated by the slow rate. In another four cases the sum of $S_{\mathrm{V} 1}$ and $R_{\mathrm{V} 6}$ fell from 86 to $50 \mathrm{~mm}$., 50 to $39 \mathrm{~mm}$., 65 to $45 \mathrm{~mm}$., and 46 to $40 \mathrm{~mm}$. respectively, and in the final case there was no change in the pre-operative total of $39 \mathrm{~mm}$., a figure only slightly above normal. other series (Stuckey, 1955), and there is no record of neonatal asphyxia, which has recently been incriminated (Record and McKeown, 1953, 1955). Information concerning this was, however, only obtained from the mother and it is not unlikely that the neonatal hospital records of these children might reveal a different picture as the mothers would not necessarily have known that asphyxia had occurred.

Incidence. The condition is not uncommon and formed $16 \%$ of the first 1,200 cases referred for suspected heart disease.

Clinical Diagnosis. Although the patent ductus probably gave rise to symptoms in about one-third 
of cases, the majority of children were referred to the heart clinic on account of the cardiac murmur.

Physical Signs. A classical Gibson murmur characteristic of patent ductus arteriosus, usually with a thrill, was present in $90 \%$ of our cases. In these patients the diagnosis was considered to be established by this physical sign alone and further examination, electrocardiography and radiology were completed mainly to exclude associated cardiac abnormalities and to elicit evidence of pulmonary hypertension which might require investigation by cardiac catheterization. In a few of these cases the murmur varied considerably from time to time, especially in respect of its loudness during diastole. This phenomenon is well recognized in patent ductus arteriosus and may be partly due to variation in the position of the heart affecting the relations between the ductus and great vessels and therefore the volume of the shunt. In one case at operation it was found that the characteristic thrill over the pulmonary artery was only present when the apex of the heart was lifted by the surgeon's hand and the long, narrow ductus, which lay very obliquely between the aorta and pulmonary artery, became more transverse and, probably, more patent. Under normal circumstances the degree of distension of the stomach and posture of the child might act in a similar manner. The murmur was less typical, in that it did not extend throughout diastole, in seven cases all with pulmonary hypertension, but the crescendo in late systole running up to an accentuated second sound and immediately followed by a softer fading diastolic murmur was very suggestive of a patent ductus. In this type of murmur and in still less typical murmurs, phonocardiography may be helpful in revealing their characteristics, but it was not used to any extent in this series. The murmur and thrill of an aortopulmonary fistula may be similar to that of a patent ductus and it is of interest that we have not seen an example of this condition in this series, whereas in some published series additional patients with such fistulae have been reported.

An exceptionally loud second sound in the pulmonary area indicated pulmonary hypertension in seven patients, in some of whom the sound was only just split or single, due probably to synchronous closure of aortic and pulmonary valves. In many of the other cases, as has already been stated, we found it difficult to decide whether a loud second sound was within the limits of normal or not. A soft pulmonary sound would, of course, throw doubt on the presence of a patent ductus or suggest an associated pulmonary stenosis.

A mid-diastolic murmur was heard in the mitral area in a high proportion of cases once its frequency had been appreciated, and it is of interest that its incidence bore a closer relationship to radiological evidence of increased size of the heart and left ventricle, to hilar enlargement and to pulmonary hyperaemia than any other physical sign. It is ascribed to the increased flow through the mitral valve which results from augmentation of the pulmonary blood flow by the volume shunted through the ductus, and will be most easily heard when the flow is large. Occasionally it is accompanied by a diastolic thrill.

A definitely collapsing pulse and wide pulse pressure suggested, on the whole, a large rather than a small patent ductus, while in many of the children a normal blood pressure and pulse were found in the presence of a normal or medium-sized ductus. A normal pulse and/or pulse pressure were, however, also found in six of the 11 cases with a large ductus complicated by pulmonary hypertension.

Radiography. The radiological findings in these children were similar to those in other published series. They were, on the whole, more marked when the ductus was large and the changes following operation were also more striking after the closure of a large ductus. In some cases reversion towards normal appearances was progressive for 18 months or longer, but details of these later films are not included in this paper. As might be expected, there was less change in those cases in which closure of the ductus was known to be incomplete or recanalization had occurred.

Electrocardiography. The electrocardiographic evidence of left ventricular dominance which was found in over a third of our patients throws light on the nature of the cardiac enlargement which occurs with persistent patency of the ductus arteriosus. The characteristics of 'diastolic overload" of the left ventricle, suggesting simple dilatation of that chamber without hypertrophy (SodiPallares and Marsico, 1955; Landtman, 1954), were found in 33 cases, while evidence of "systolic overload was only seen in two. The latter type of record is found with muscular hypertrophy of the left ventricle which, in cases of patent ductus arteriosus, is said to be due usually to associated systemic hypertension, but in these two the blood pressure was normal, being $110 / 75$ and $105 / 85 \mathrm{~mm}$. $\mathrm{Hg}$ respectively. However, the E.C.G. in both cases reverted to normal soon after operation and it seems unlikely that the ventricular muscle could lose its hypertrophy so rapidly. Mannheimer (1948) demonstrated that a large number of patients with 
patent ductus arteriosus showed electrocardiographical evidence of coronary insufficiency after exercise and it is possible that inadequate diastolic filling of the coronary arteries causes the inversion of the $T$ wave and depression found in these cases. In this event, rapid reversion of the E.C.G. to normal would be expected after surgical closure of the ductus.

Conclusive evidence of right ventricular hypertrophy in a case of patent ductus arteriosus is of considerable importance. Electrocardiography suggested this change in 14 of the 83 cases fully examined, if one accepts $10 \mathrm{~mm}$. as the upper limit of normal for $R_{\mathbf{v} 1}$ (Switzer and Besoain, 1950). The lack of evidence of pulmonary hypertension in some of these children and its occurrence in some of those with a normal E.C.G., however, illustrates the need for caution in interpreting right ventricular dominance in these cases, especially in young children, in whom a variable degree of this pattern is normally found; and it is possible that in lead $V_{1}$ an $R$ wave of 13 to $15 \mathrm{~mm}$. or even more may on occasion be unassociated with right ventricular hypertrophy and therefore within the limits of normal. However, a study of our more recent cases suggests that greater attention should in future be paid to lead $V_{3 R}$ which appears to give more consistent evidence of right ventricular change than lead $V_{1}$. This is in line with the recent observation that $\mathrm{V}_{4 \mathrm{R}}$ in adults gives the most reliable information concerning the right ventricle (Camerini, Goodwin and Zoob, 1956).

Cardiac Catheterization. As stated above, this method of investigation was employed in a few cases in this series only. In future it should be necessary even less frequently, as the condition will probably be diagnosed in the majority of children at the latest on school entry at the age of 5 years when it will only occasionally be complicated by significant pulmonary hypertension. In some cases, however, it will still be necessary in order to establish the diagnosis and the direction of the shunt when this is in doubt.

In view of the technical difficulties that may be encountered in closing a large ductus, it is helpful to the surgeon if the size of the vessel can be predicted before operation. The data from this series which might have been useful in achieving this aim are set out in Table 6, which compares the findings in large and small ductuses, omitting those of intermediate size.

It will be seen that there is a rough correlation between the clinical findings and the external diameter of the ductus. That the correlation is not
TABLE 6

RELATION OF FINDINGS TO SIZE OF THE PATENT DUCTUS

\begin{tabular}{|c|c|c|}
\hline Findings & $\begin{array}{l}\text { Patent Ductus } \\
\text { up to } 7 \mathrm{~mm} \text {. }\end{array}$ & $\begin{array}{l}\text { Patent Ducrus } \\
\text { over } 10 \mathrm{~mm} \text {. }\end{array}$ \\
\hline 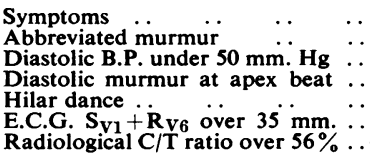 & $\begin{array}{l}25 \% \\
\mathrm{Ni1} \\
22 \% \\
20 \% \\
14 \% \\
32 \% \\
22 \%\end{array}$ & $\begin{array}{l}86 \% \\
43 \% \\
61 \% \\
82 \% \\
50 \% \\
69 \% \\
72 \%\end{array}$ \\
\hline
\end{tabular}

closer is not surprising, as one would expect that the clinical findings would depend more on the blood flow through the ductus than upon its external diameter and it has shown that there is no direct relationship between these two (Fowler and Mannix, 1955; Taylor, Pollack, Burchell, Clagett and Wood, 1954). Two variable factors which may account for this are the thickness of the wall of the ductus and the angle at which it joins the pulmonary artery. While, therefore, it is impossible from clinical findings alone to predict accurately the size of a ductus, one may with a fair degree of certainty suspect the presence of the unusually large vessel which frequently gives rise to difficulties at operation. Pulmonary hypertension may also give rise to difficulty at operation and should be diagnosed beforehand. Its presence will be suspected when the pulmonary second sound is loud and closely split or single and when there are physical signs, E.C.G. changes and radiological appearances of right ventricular hypertrophy. In addition, the peripheral pulmonary plethora may be reduced by arteriolar constriction so that the outer parts of the lung fields appear light while the hila remain engorged. In all such cases the pulmonary artery pressure must be measured and the direction of the shunt established by cardiac catheterization.

Treatment. Policy with regard to treatment must depend first upon our knowledge of the outcome to be expected in untreated cases. Shapiro and Keys (1943) expressed the situation clearly when they wrote that patent ductus arteriosus is a time bomb with a long fuse but still a bomb. They found that $80 \%$ of patients with patent ductus arteriosus eventually died from the effects of it and that the expectation of life was only 35 to 40 years. Campbell (1955) reviewed 20 patients still alive at 35 years or more. Nine were losing ground by the age of 42 at the latest and a further six showed marked E.C.G. and radiological evidence of cardiac enlargement. In addition to a shorter expectation of life, there is commonly a reduction of the cardiac reserve which leads eventually to congestive cardiac failure; 
pulmonary hypertension develops in many cases and results in a reversal of the shunt through the ductus with clanose tardive (Gross, 1952); and infective endocarditis may supervene. These complications are seldom seen in childhood but they present an unpleasant prospect for the untreated adult. During childhood growth may be limited (Porter, 1947; Muir and Brown, 1932), and was seen as a general phenomenon in this series, but the weights and heights recorded were not considered to be sufficiently accurate for detailed analysis.

While spontaneous closure of a patent ductus arteriosus has been reported, in exceptional cases as late as 34 years of age, its rarity does not justify the expectation that it will occur in any individual case (Brown, 1950; Campbell, 1955).

It is therefore desirable that every patent ductus diagnosed during childhood, in which the shunt is from left to right, should be closed surgically. This practice now has wide support, even when the aortic and pulmonary artery pressures are almost balanced (Bonham Carter, 1954). It is, however, essential that such operative treatment must be as nearly as possible devoid of risk if it is to be recommended universally as a prophylactic measure in children, many of whom are without symptoms. In this series no patient died following ligation of a patent ductus arteriosus. The single death was in the child with advanced pulmonary hypertension and reversal of shunt under the conditions of the operation, whose ductus was not ligated, in conformity with the view that ligation in such cases usually proves fatal. Twenty-three per cent. of our patients were under 4 years of age and $66 \%$ under 7 years when submitted to surgery. The procedure was tolerated well by the great majority, especially by the toddlers, many of whom seemed to be in normal health 48 hours after the operation. It has therefore become our practice in all straightforward and uncomplicated cases to advise closure of a patent ductus as soon as it is convenient.

The relief of symptoms, and the radiological and electrocardiographic evidence of decrease in the size of the heart in this series following operation indicate the possible reversibility, at any rate during childhood, of the cardiac changes caused by patency of the ductus, and support the policy of early closure. These findings are in agreement with those of Mannheimer (1948) who used an elaborate radiological technique for estimating the heart volume in 14 cases and found that ligation of a patent ductus arteriosus was followed by a decrease in volume in 12.

The good functional result indicated by this evidence was seen in the majority of cases. In an appreciable number of such patients, however, persistence or recurrence of a typical murmur suggested incomplete closure, or recanalization of the ductus. In this respect our experience of simple ligation of a ductus is similar to that of others (Sellors, 1948; Potts et al., 1949; Gilchrist, 1948; Shapiro and Johnson, 1947), namely, that it will not always accomplish complete and permanent obliteration of the vessel. Although incomplete closure or recurrence are usually not accompanied by a shunt as large as before operation and are not, therefore, so liable to result in cardiac failure, nevertheless the possibility of infective endocarditis persists and a second attempt at closure is advisable, unless the surgeon considers the danger of further interference to be too great. It was for this reason that the more difficult operation of division of the ductus was adopted by Gross and others (Gross, 1952 ), even though the mortality rate of the procedure may be $2 \%$ or more in the best hands.

Summary

One hundred and eight children treated surgically for patent ductus arteriosus have been reviewed. The results of operation are analysed and the diagnosis of the condition is discussed.

We are grateful to Mr. F. Ronald Edwards and to Mr. B. J. Bickford for the surgical treatment of these patients and for the operative findings; to Dr. G. J. Rees for the anaesthetics; to Dr. Nora Walker and Dr. J. K. Walker for the radiological findings; to Dr. R. E. Rewell and his laboratory staff for the estimations of blood oxygen saturation; and to Mr. E. J. Caldwell and Mr. N. Clark for the electrocardiograms. We also wish to thank Professor Norman Capon for helpful criticism.

\section{REFERENCES}

Bonham-Carter, R. E. (1954). In Recent Advances in Paediatrics, ed. Gairdner, D., p. 402 . London.

Brown. J. W. (1950). Congenital Heart Disease, 2nd ed. London. Camerini. F., Goodwin, J. F. and Zoob, M. (1956). Brit. Heart J., 18. 13 .

Campbeli M. (1955) Ibid 17, 511.

Fowler, N. O. and Mannix, E. P. (1955). J. clin. Invest., 29, 745.

Fowler, N. O. and Mannix, E. P. (1955).

Gikhrist, R. (1948). Brit. Heart J., 10, 75.

(1952). Amer. J. Med., 12, 472.

and Hubbard, J. P. (1939). J. Amer. med. Ass., 112, 729.

Landtman, B. (1954). Circulation, 10, 871.

Lind. J. (1950). Acta radiol. (Stockh.), Suppl. 82

Mannheimer, E. (1948). Acta paedit. (U'Ppsala), 35. Suppl. 1, p. 217.

Meyer, R. R. (1949). Radiology, 53, 363.

Meyer, R. R. (1949). Radiology, 53, 363 .
Muir, D. C. and Brown, J. W. (1932). Archives of Disease in Childhood, 7, 291 .

Orme, H. W. and Adams, F. H. (1952). J. Pediat., 41, 53

Porter, W. B. (1947). Amer. J. med. Sci., 213, 178.

Potts, W. J.. Gibson, S., Smith, S. and Riker, W. L. (1949). Arch. Surg. (Chicago), 58. 612 .

Record, R. G. and McKeown, T. (1953). Brit. Heart J.. 15. 376.

Record, R. G. and McKeown, T. (1953).

Scott, H. W. (1950). Surg. Gynec. Obstet., 90, 91

Sellors, H. (1948). Brit. Heart J., 10, 76.

Shapiro, M. J. and Johnson, E. (1947). Amer. Heart J., 33, 725.

- and Keys, A. (1943). Amer. J. med. Sci., $206,174$.

Sodi-Pallares, D. and Marsico, F. (1955). Amer. Heart J., 49, 202.

Starer, F. (1953). Brit. med. J., 1. 971.

Stuckey, D. (1955). Med. J. Aust., 1, 749.

Switzer, J. L. and Besoain, M. (19S0). Amer. J. Dis. Child., 79, 449.

Taylor, B. E., Pollack, A. A., Burchell, H. B., Clagett, O. T. and Wood, E. H. (1950). J. clin. Invest., 29, 745.

Tubbs, O. S. (1755). Brit. med. Bull., 11, 200. 